\title{
Cluster EDI convection measurements across the high-latitude plasma sheet boundary at midnight
}

\author{
J. M. Quinn ${ }^{1}$, G. Paschmann ${ }^{2}$, R. B. Torbert ${ }^{1}$, H. Vaith $^{2}$, C. E. McIlwain ${ }^{3}$, G. Haerendel ${ }^{4}$, O. Bauer ${ }^{2}$, T. M. Bauer ${ }^{2}$, \\ W. Baumjohann ${ }^{5}$, W. Fillius ${ }^{3}$, M. Foerster ${ }^{2}$, S. Frey ${ }^{2}$, E. Georgescu ${ }^{2}$, S. S. Kerr ${ }^{3}$, C. A. Kletzing ${ }^{6}$, H. Matsui ${ }^{1}$, \\ P. Puhl-Quinn ${ }^{2}$, and E. C. Whipple ${ }^{7}$ \\ ${ }^{1}$ Space Science Center, Morse Hall University of New Hampshire Durham, NH 03824, USA \\ ${ }^{2}$ Max-Planck-Institut für extraterrestrische Physik D-87545 Garching, Germany \\ ${ }^{3}$ Center for Astrophysics and Space Sciences University of California, San Diego La Jolla, CA 92093, USA \\ ${ }^{4}$ International University Bremen, 28725 Bremen, Germany \\ ${ }^{5}$ Space Research Institute, 8042 Graz, Austria \\ ${ }^{6}$ Department of Physics and Astronomy University of Iowa Iowa City, IO 52242, USA \\ ${ }^{7}$ Geophysics Program University of Washington Seattle, WA 98195, USA
}

Received: 17 April 2001 - Revised: 1 July 2001 - Accepted: 6 July 2001

\begin{abstract}
We examine two crossings of three Cluster satellites from the polar cap into the high-latitude plasma sheet at midnight local time, using data from the Electron Drift Instrument (EDI). EDI measures the full electron drift velocity in the plane perpendicular to the magnetic field for any field and drift directions. The context of the measured convection velocities is established by their relation to the intense enhancements in $1 \mathrm{keV}$ electrons, also measured by EDI, as the satellites move from the polar cap into the plasma sheet boundary. In both cases presented here, the cross $\boldsymbol{B}$ convection in the polar cap is anti-sunward (toward the nightside plasma sheet) with a small duskward component. As the satellites enter the plasma sheet boundary region, the dawndusk convective flow component reverses its sign, and the flow in the meridianal plane (toward the center of the plasma sheet) drops substantially. The relatively stable convection in the polar cap becomes highly variable as the PSBL is encountered. The timing and sequence of the boundary crossings by the Cluster satellites are consistent with a relatively static structure on a time scale of the few minutes in satellite separations. In one of the two events, the plasma sheet boundary has a spatially separate structure that is crossed by the satellites before entering the plasma sheet.
\end{abstract}

Key words. Magnetospheric physics (electric fields; magnetopause, cusp and boundary layers; instruments and techniques)

\section{Introduction}

A primary objective of the Cluster mission is the investigation of boundary structures using multi-point measurements to deconvolve spatial and temporal variations. The bound-

Correspondence to: J. M. Quinn (jack.quinn@unh.edu) ary between the high-latitude plasma sheet and the polar cap/tail lobe is one such region to be explored by Cluster with north-south traversals at distances of $\sim 7 R_{E}$ near perigee and $\sim 20 R_{E}$ at apogee. In this paper, we present initial results from two consecutive Cluster perigee passes through the polar cap plasma sheet boundary at midnight, using electron drift velocities and $1 \mathrm{keV}$ electron fluxes from the Electron Drift Instrument (EDI).

The high-latitude nightside plasma sheet boundary region is home to a wide range of important processes involved in auroral acceleration, ionospheric outflow, magnetic reconfiguration, transient convective plasma transport, and electromagnetic energy transfer. The electric fields and resulting convective flows, together with other related manifestations of the complex physical processes at the plasma sheet boundary, have been investigated on several missions (ISSE, S33, Interball, Polar), (e.g. Cattell et al., 1982; Eastman et al., 1984; Pederson et al., 1985; Torbert and Mozer, 1978; Sauvaud et al., 1999; Wygant et al., 2000).

Cluster EDI measurements at the plasma sheet boundary region offer multi-point measurements of the electron drift velocities, or equivalently the electric fields, for both components of the convective drift, for arbitrary orientations of the magnetic field with respect to the spacecraft spin axis. We present below a first look at EDI measurements in the nightside high-latitude plasma sheet boundary region. Results on the dayside are described in a companion paper (Paschmann et al., 2001, this issue).

\section{Technique}

The Electron Drift Instrument measures the electron drift velocity, $\boldsymbol{V}_{d}$, by detecting the displacement of two weak beams of test electrons. The technique intrinsically measures both components of drift in the plane perpendicular to the ambi- 
ent magnetic field, $\boldsymbol{B}$, for arbitrary orientations of $\boldsymbol{V}_{d}$ and $\boldsymbol{B}$ with respect to the spacecraft spin axis.

The physical basis of the measurement is the perturbation of the electron's gyro orbit from the circular trajectory that it would follow in the absence of a drift. This perturbation can be measured in two different ways in order to determine the magnitude and direction of the electron drift velocity. The first method uses the fact that the perturbed trajectory of an electron beam, fired perpendicular to the ambient magnetic field, returns to the spacecraft after one or more gyro orbits only when fired in unique directions. By finding these directions, one can deduce the drift velocity using a "triangulation" technique. The second method measures the timesof-flight for the electron beams to return to the spacecraft, which in the presence of a drift, differ from the gyro period by amounts that are proportional to $\pm V_{d}$. The two techniques are complementary in that triangulation is most accurate for small-to-moderate drifts, while the time-of-flight is better for moderate-to-large drifts. Cluster's time-of-flight measurements are discussed further in the companion paper by Paschmann et al. (2001, this issue). In this paper, we present data using only the triangulation technique.

\subsection{EDI triangulation measurement of drift velocity}

In a uniform magnetic field with no other forces acting, an electron beam fired in any direction perpendicular to the ambient magnetic field, $\boldsymbol{B}$, will execute a circular gyro orbit and return to its starting point. In the presence of a transverse electric field, $\boldsymbol{E}$ (or magnetic gradient), the trajectories are distorted, and the electrons no longer return to their starting position after one gyro period. Instead, all electrons starting at a particular location are displaced by the same amount in one gyro period due to the $\boldsymbol{E} \times \boldsymbol{B}$ (or $\operatorname{grad}-\boldsymbol{B}$ ) drift, regardless of their original direction of travel. This displacement is independent of beam energy for the $\boldsymbol{E} \times \boldsymbol{B}$ drift, and proportional to the energy for the grad- $B$ drift. The drift step, $\boldsymbol{d}$, is defined to be the net displacement during one gyro period, $T_{g}$, due to a drift velocity, $\boldsymbol{V}_{d}$ (Quinn et al., 1979)

$\boldsymbol{d}=\boldsymbol{V}_{d} T_{g}$

A measurement of the drift step, together with the knowledge of the gyro period, is thus sufficient to determine the drift velocity.

The EDI triangulation technique is illustrated conceptually in Fig. 1, which is drawn in the plane perpendicular to $\boldsymbol{B}$ (the “ $\boldsymbol{B}_{\perp}$ plane"). There are two electron guns, with a detector (Det) located halfway in between. Red and blue lines represent the electron beams. The electron gyro radius is very large $(\geq 1 \mathrm{~km})$ compared to the scale of the figure $(\sim 1 \mathrm{~m})$, so the gyro trajectories of the beams appear as straight lines. The magnitude and orientation of the drift step, $\boldsymbol{d}$, is determined by the ambient fields, as described above. We have drawn $\boldsymbol{d}$ with its head positioned at the detector. By definition of the drift step, any $90^{\circ}$ pitch-angle electron passing through the tail end of $\boldsymbol{d}$ will be displaced by the drift velocity and will hit the detector exactly one gyro period later.

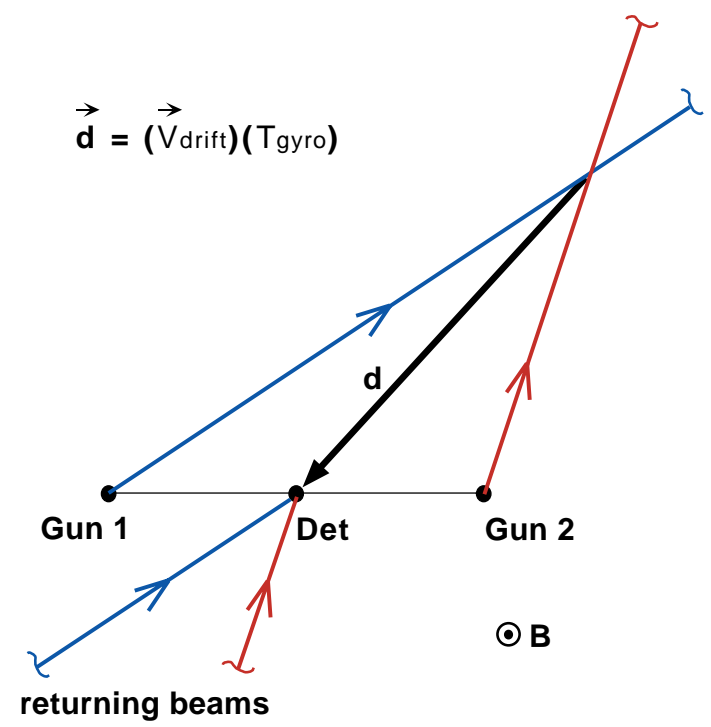

Fig. 1. Principle of EDI triangulation, projected in the plane perpendicular to $\boldsymbol{B}$. The gyro radius of the beam electrons is much larger than the scale of the figure, so the beams appear as straight lines. The "drift step", $\boldsymbol{d}$, is the gyro motion displacement in exactly one gyro period. With the head of the $\boldsymbol{d}$ vector placed at the detector, electron beams successfully aimed at the tail of $\boldsymbol{d}$ will return to the detector after one gyro orbit. The combined firing directions of two guns and the known geometric positions of the guns and detector are sufficient to determine the drift step, and thus the drift velocity, from triangulation.

Therefore, an electron beam aimed so that it passes through the tail of $\boldsymbol{d}$ will return to hit the detector after one gyro orbit. Thus, the tail of $\boldsymbol{d}$ represents a "target", which if hit, will result in the electrons returning to the detector. Electrons fired in other directions and not passing through the target position will not hit the detector, except for those fired in the opposite direction, which also have a virtual source at the tail of $\boldsymbol{d}$.

When both guns are aimed in the unique directions that allow their beams to hit the detector after one gyro orbit, one can determine from triangulation the beam intersection point, which corresponds to the tail of $\boldsymbol{d}$. Using the known locations of the guns and the detector, one can then calculate the vector $\boldsymbol{d}$. Together with the knowledge of the gyro period, this measurement of $\boldsymbol{d}$ determines the drift velocity of the electrons.

The Cluster EDI gun-detector configuration necessarily differs from that shown in Fig. 1, but it is geometrically equivalent. (The Cluster geometry, together with the full gyro motion of both electron beams, is illustrated in Fig. 1 of Paschmann et al., 2001, this issue). There are two gundetector units (GDUs) mounted on opposite sides of the spacecraft, with oppositely directed fields-of-view. Each gun is capable of firing its beam in any direction within a bit more than a full hemisphere, at polar angles up to $96^{\circ}$, to an accuracy of better than $1^{\circ}$. Similarly, each detector is able to detect electrons coming from any selected direction within a hemispherical field-of-view for polar angles up to $100^{\circ}$. The beam fired by each gun is received by the detector on 


\section{Cluster-EDI 20 Oct. $2000 \quad$ SC-3}

\section{$0.5 \mathrm{~s}$ Intervals at 10 Minute Spacing}

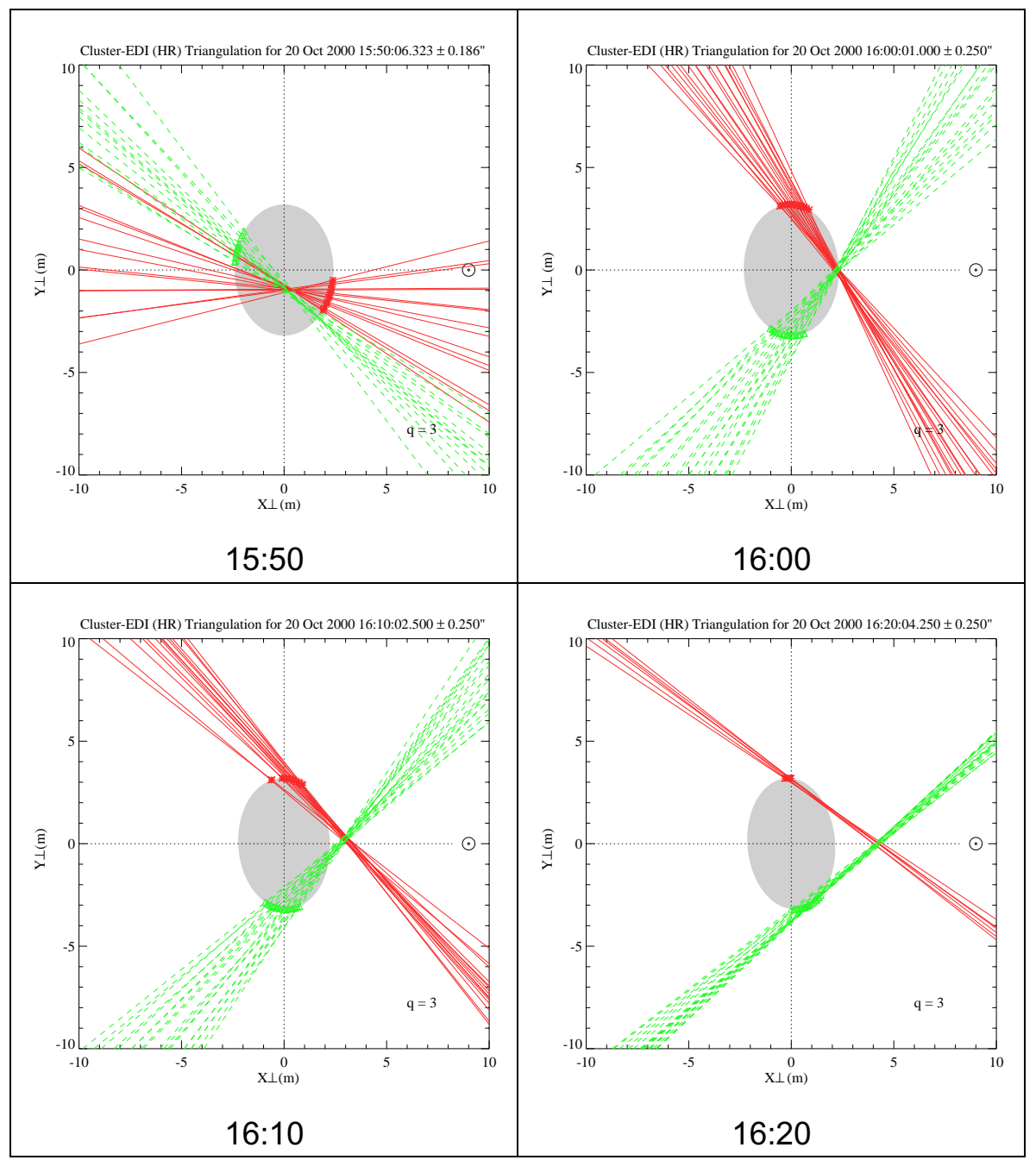

Fig. 2. Sample EDI triangulation plots for four $0.5 \mathrm{~s}$ intervals, spaced $10 \mathrm{~min}$ apart. Red and green lines are the firing lines of the two electron guns for those times when the beams were properly aimed to return to the detector. The gun positions (symbols) projected into the plane perpendicular to the magnetic field move on an ellipse as the spacecraft rotates through 1/8 of a spin in each panel. The measured "drift step" is the vector from the beam intersection to the center of the figure.

the opposite side of the spacecraft. Thus, for the purposes of triangulation, the baseline separating a gun from its corresponding detector is the spacecraft diameter projected into the $\boldsymbol{B}_{\perp}$ plane. At any instant, one gun fires at a detector that is displaced from it in the $\boldsymbol{B}_{\perp}$ plane by a baseline $\boldsymbol{b}$, while the other gun fires at a detector displaced by $-\boldsymbol{b}$. Therefore, the Cluster configuration is geometrically identical to that shown in Fig. 1 of this paper, where each of the two guns is separated from its detector by equal distances in opposite directions. (The triangulation baseline, formed by the projection of the gun-detector separation vectors in the $\boldsymbol{B}_{\perp}$ plane, varies with the orientation of the ambient magnetic field and spacecraft spin phase.) For the purposes of displaying triangula- tion data, we will continue to use this picture, i.e. two guns and a single "virtual detector" located halfway in between at a distance of one spacecraft diameter from each gun.

Figure 2 shows sample EDI triangulation data in four, $0.5 \mathrm{~s}$, intervals, separated from each other by $10 \mathrm{~min}$. In each interval, those beam-firing directions that returned to the detector are plotted and drawn as straight lines with red and green indicating the beams from the two different guns. The position of the virtual detector is at the center of the picture. As the satellite rotates, the projection of the gun positions sweep out an ellipse when projected into the $\boldsymbol{B}_{\perp}$ plane. Shading indicates this ellipse, and the gun firing positions at the time of each return beam (each "hit") are shown by small 
CL1 23 Feb 2001 10:30-18:30
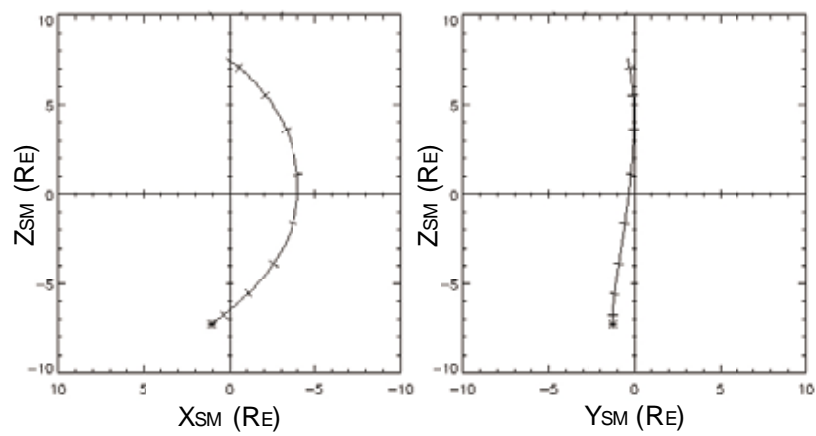

CL1 21 Feb 2001 01:00-09:00
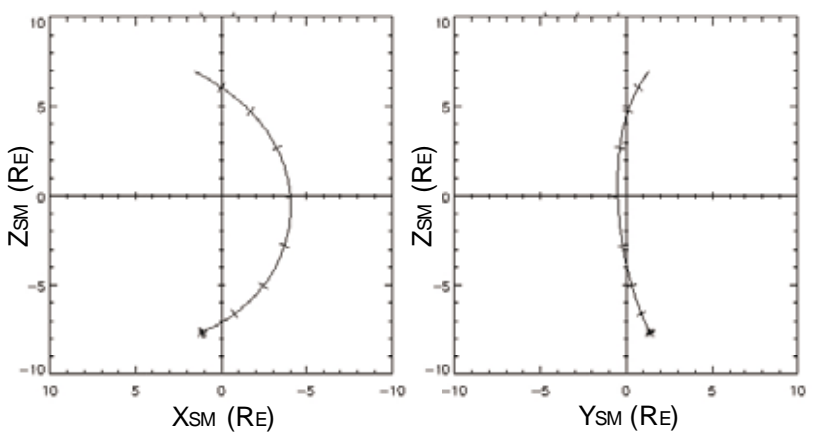

Fig. 3. Cluster 1 orbit plots in solar magnetospheric coordinates for perigee passes on 23 February (top) and 21 February (bottom) 2001. The Cluster satellites approach perigee through the southern polar cap, pass through the equatorial plane near midnight, and exit to the north. The 8-h orbit plots begin at the asterisk, with tics placed at each UT hour, on the hour.

symbols where the beams intersect the edge of the ellipse. In each of the four time periods, the beams from both guns cross at a well-defined intersection, defining the location of the target. The drift step is simply the vector from this target to the virtual detector at the center of the figure. During this 30-min period from which these samples are taken, the drift step changes direction by approximately $90^{\circ}$ and increases steadily in magnitude.

The ground analysis of the beam firing directions needed to determine the drift steps, and ultimately the drift velocities, uses essentially the same data as displayed in Fig. 2. A fitting procedure identifies the best beam intersection point for all successful beams within a chosen analysis interval, and assigns a chi-squared "quality" to the fit for each interval. This analysis is described in the companion paper by Paschmann et al. (2001, this issue). The results presented in this paper use analysis intervals of one-half spin $(2 \mathrm{~s})$. However, the analysis can be done over arbitrary intervals, providing that enough beams are present to define a clear intersection. The limiting time resolution is not fixed, but depends on the rate of successful beam "hits" that are obtained by the onboard tracking algorithm.

\subsection{Cluster EDI implementation}

The electron beam triangulation technique was first developed by the group at the Max-Planck-Institut für extraterrestrische Physik (MPE), and flown on the GEOS-2 satellite (Melzner et al., 1978). While the GEOS experiment clearly established the viability of the measurement technique (e.g. Baumjohann et al., 1985), its time resolution was limited to the 6-s spacecraft spin period, and its beam deflection capability restricted operation to a narrow range of magnetic field orientations. The EDI instrument for the Cluster and Equator-S missions was developed to remove these limitations as well as to incorporate the time-of-flight technique and other improvements that were necessary to accommodate the wide range of anticipated magnetic fields, electric fields, and ambient (background) electron fluxes.

Detailed technical descriptions of the EDI instrument are given in several previous publications (Paschmann et al., 1997, 1998, 1999; Vaith et al., 1998), with recent updates discussed by Paschmann et al. (2001, this issue). The key elements for the present paper are the beam firing angles of the guns, the beam recognition by the detector, and the beam acquisition and tracking algorithms which allow for sufficiently frequent "hits" to obtain good triangulation measurements. The angles in the triangulation measurement are taken from the gun firing directions, which were calibrated prior to launch over the entire gun solid angle. Since the beam can only return to the spacecraft if it is fired within approximately $1^{\circ}$ (the beam width) of the $\boldsymbol{B}_{\perp}$ plane, we can check and update these calibrations in orbit by analyzing the distribution of beam hits over the gun solid angle for various magnetic field orientations. On Cluster and Equator-S, we have found that the guns' ground calibrations perform very well in orbit.

Each EDI gun-detector pair independently acquires and tracks the target by controlling the gun firing directions with a sophisticated, onboard servo algorithm. EDI on Cluster uses beam energies of 1.0 and $0.5 \mathrm{keV}$ to distinguish the effects of $\boldsymbol{E} \times \boldsymbol{B}$ drifts, which are independent of energy, and grad- $B$ drifts, which are proportional to energy. In most regions, the $\boldsymbol{E} \times \boldsymbol{B}$ drift is strongly dominant, and thus the differences between the 1.0 and $0.5 \mathrm{keV}$ beams are quite small (see, for example, Paschmann et al., 2001, this issue). In this paper, we present only results from $1 \mathrm{keV}$ beams.

The electron beams are amplitude-modulated with one of two pseudo-noise codes: a "long code" with 127 chips, or a "short code" with 15 chips, and the detected counts are processed by a 15-channel correlator. To acquire the target, the beam firing direction is stepped at a constant angular rate in the $\boldsymbol{B}_{\perp}$ plane until the beam-recognition algorithm records a hit by analyzing the counts from the different correlator channels. When the beam is detected, the onboard tracking algorithm reverses the angular stepping direction so that the beam is swept repeatedly back and forth across the target direction.

The relevant physical parameters for the EDI triangulation technique are the magnetic field and the drift velocity, 

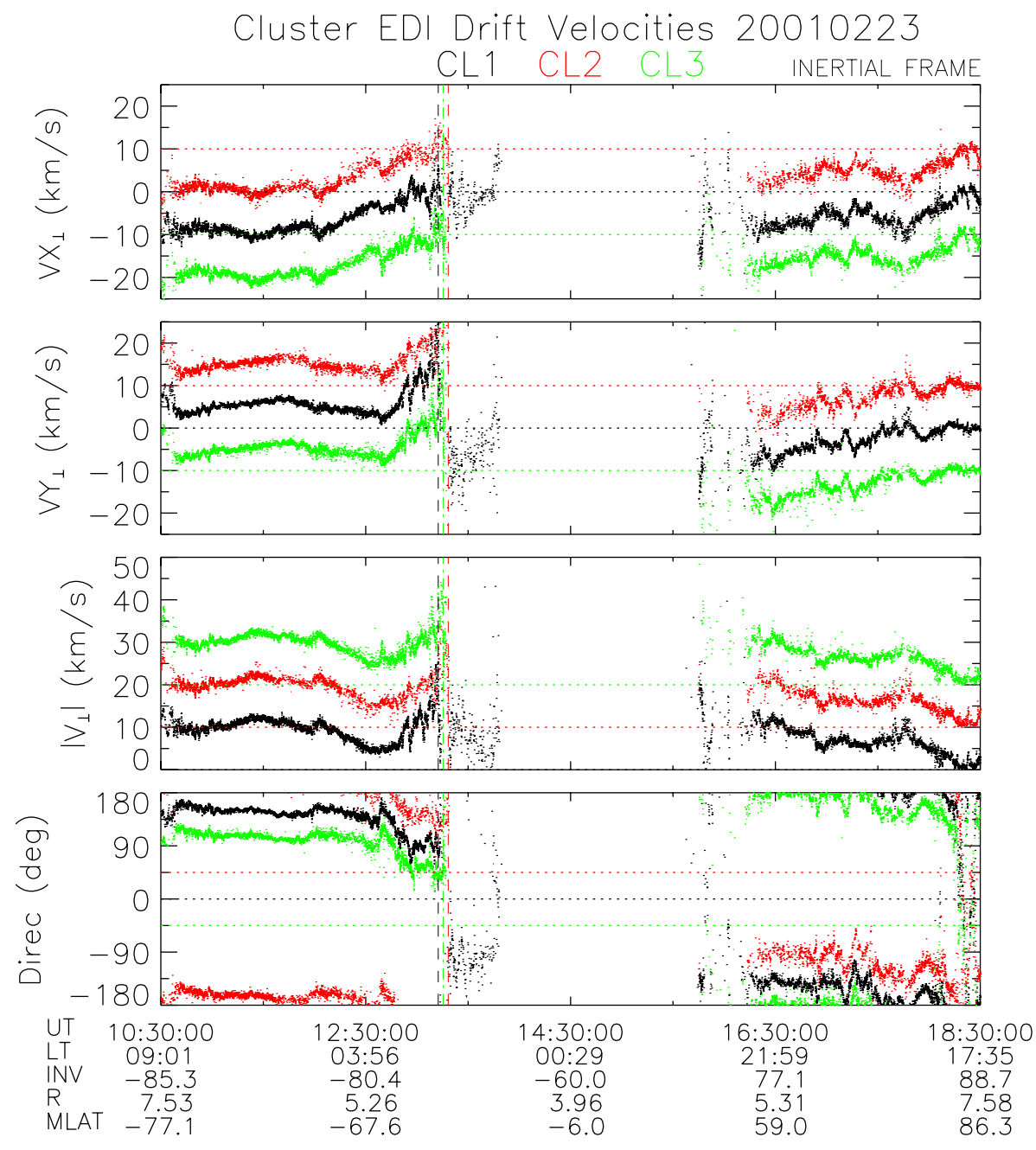

Fig. 4. EDI drift velocities for 23 February 2001, for spacecraft 1, 2, and 3, using the standard Cluster color assignments $(1=$ black; $2=$ red; and $3=$ green). $V X_{\perp}$ and $V Y_{\perp}$ are velocity components in the plane perpendicular to $\boldsymbol{B}$ with $\boldsymbol{X}_{\perp}$ closest to $\boldsymbol{X}_{\mathrm{GSE}}$ and $\boldsymbol{Y}_{\perp}$ closest to $\boldsymbol{Y}_{\mathrm{GSE}}$. Dashed vertical lines mark the satellite encounters with the $1 \mathrm{keV}$ electron boundary (see Fig. 7).

which together determine the magnitude and direction of the drift step. Details of this dependence are given in Quinn et al. (1999) and Paschmann et al. (1999). The accuracy with which one can determine the drift step through triangulation depends on several factors, including the knowledge of the beam firing direction and the relative magnitude and orientation of the gun-detector baseline $\boldsymbol{b}$, projected into the $\boldsymbol{B}_{\perp}$ plane, with respect to the drift step, $\boldsymbol{d}$. In general, triangulation is most accurate when the baseline is comparable in magnitude to the drift step. However, with firing angles known to $1^{\circ}$, the drift steps can be measured to better than $20 \%$ over a sufficient dynamic range for many magnetospheric regions. When drift steps are a few tens of times larger than the baseline, the drift direction is known accurately, but the magnitude obtained by triangulation becomes increasingly uncertain. Fortunately, as the triangulation accuracy decreases in the large $\boldsymbol{d}$ regime, the time-of-flight technique becomes more accurate (e.g. often in the magnetosheath), making the two techniques highly complementary.
For extremely small drift steps, the measured magnitude is known to be small, but the direction becomes increasingly uncertain. For the region of space covered in this paper, drift steps in the satellite frame of reference are typically of the order of $1 \mathrm{~m}$, which is ideal for triangulation with baselines on the order of the spacecraft diameter.

A key feature of EDI is that the technique is independent of the orientations of the electric and magnetic fields. The instrument measures both components of the drift velocity (or equivalently, the electric field) in the $\boldsymbol{B}_{\perp}$ plane, without regard to the relative orientations of the magnetic field, the spacecraft spin axis, and the drift direction.

We note that in the presence of a sufficiently large electric field parallel to $\boldsymbol{B}$, the beam would be accelerated along the magnetic field and would not return to the spacecraft. In principle, the detection of a return beam places an upper limit on the parallel electric field. In practice, however, the limiting value is very large, and generally does not play a role in EDI tracking nor provide a useful measurement of $\boldsymbol{E}_{\|}$. For a 

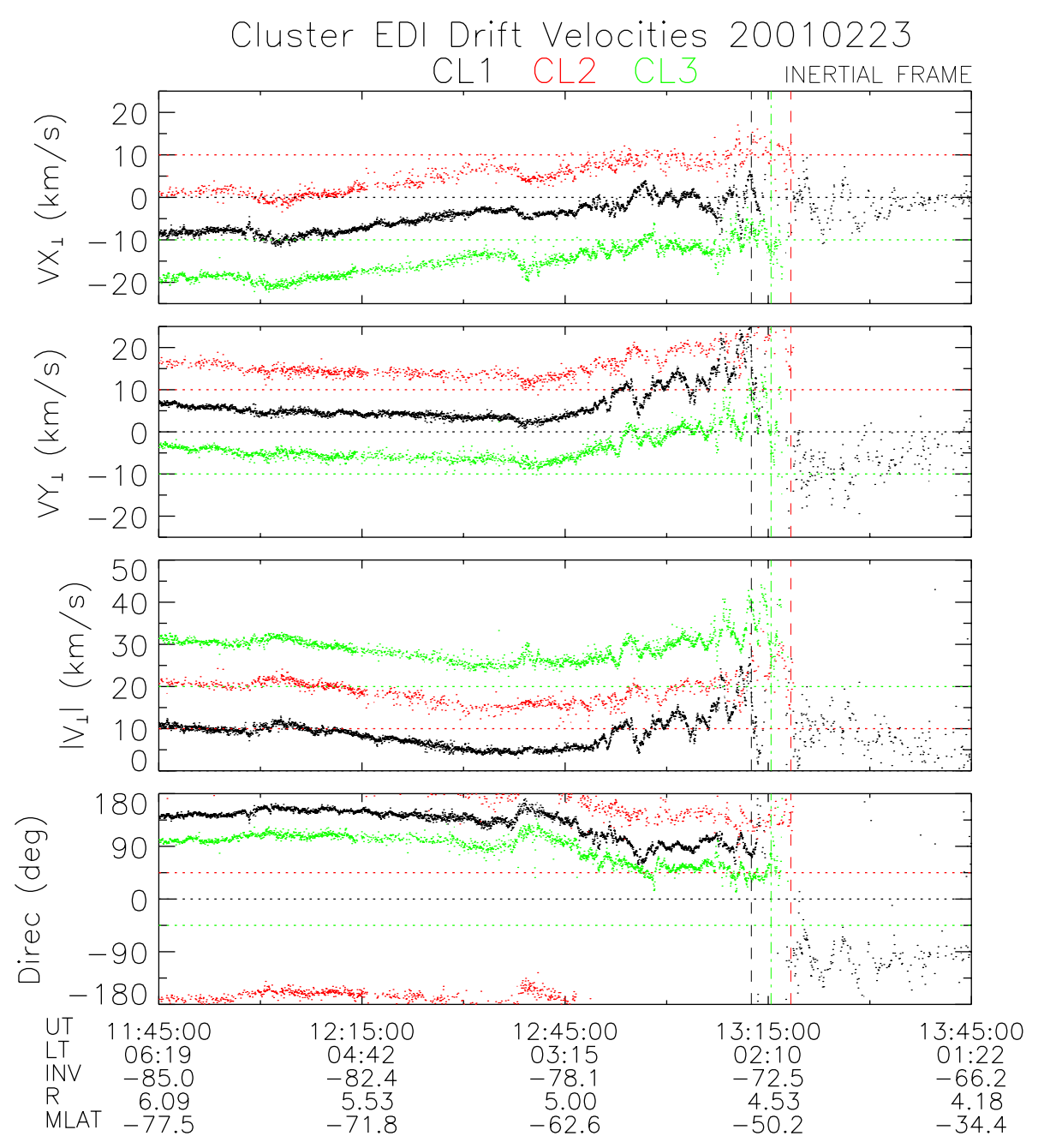

Fig. 5. Drift velocities for the 23 February southern hemisphere crossing from the polar cap into the high-latitude plasma sheet. Same format as Fig. 4. Vertical dashed lines show when the spacecraft encountered the intense $1 \mathrm{keV}$ electron boundary (see Fig. 7).

given beam width and energy, the ratio of $\boldsymbol{E}_{\|} / \boldsymbol{B}$ determines whether the displacement of the beam by the parallel electric field in one gyro orbit is greater than its dispersion along the magnetic field. As an example, a $1 \mathrm{keV}$ beam of $1^{\circ}$ halfwidth in a $100 \mathrm{nT}$ magnetic field requires a parallel electric field of $10.4 \mathrm{mV} / \mathrm{m}$ to displace the beam enough so that it is not detected.

\subsection{Ambient electron monitoring}

In addition to sensing the return electron beams, the EDI detectors make a continuous measurement of ambient electrons at the beam energy of 1.0 or $0.5 \mathrm{keV}$, and pitch-angles of approximately $90^{\circ}$. While these ambient fluxes represent a source of "background" to the primary EDI beam detection, they also provide a valuable diagnostic of the local plasma regime at relatively high time resolution. In this paper, we present ambient electron measurements at $1 \mathrm{keV}$ only.

During normal operations, the EDI detectors are continuously steered perpendicular to the magnetic field in order to sense the return beam electrons. Depending on whether or not the beam pseudo-noise code is detected by the EDI correlator, the electron counts are identified as either ambient background, or a returning beam. The time resolution of the ambient electron measurements varies with telemetry mode and with the number of samples in which the return beam is successfully detected. For the normal-rate telemetry data used in this paper, the electron count rate sampling is returned at a rate of $8 \mathrm{samples} / \mathrm{s}$. (In high-rate telemetry, the sampling is 8 times faster). Depending on the onboard beam-tracking duty cycle, the division of these samples between ambient and beam measurements can vary substantially, from nearly $100 \%$ beam during good tracking, to less than $20 \%$. Assuming that typically $50 \%$ of the telemetered electron samples are beams, then the ambient electron data have an average sampling rate of approximately 4 samples/s.

The EDI geometric factor and efficiencies vary somewhat with the detector look-direction and, more substantially, with the detector "Optics State" (see Paschmann et al., 1997, for a discussion of the adjustable geometric factor of EDI's de- 
tector optics). The ambient electron data presented here are from a fixed Optics State. As is evident in the data, the effects of the look-direction are negligible compared to the ordersof-magnitude variations in the ambient electrons which are presented below. In future work, a quantitative on-orbit determination of the detector geometric factor and efficiencies will enable smaller scale ambient electron features to be reliably used for detailed timing.

\section{Convection measurements at high-latitudes near perigee}

We now examine EDI convection velocities in relation to the high-latitude plasma sheet boundary during two consecutive perigee passes of the Cluster spacecraft in February 2001. At this time, the orbit plane was nearly in the noon-midnight meridianal plane, with perigee at approximately midnight. Figure 3 shows the Cluster orbits for the 8-h perigee passes on 23 and 21 February, in solar magnetospheric coordinates. For each pass, we will present an overview of the 8-h period, and then look in detail at the southern hemisphere crossings from the polar cap into the plasma sheet. During the orbits of this study, EDI was not operated for approximately a 2-h period centered on perigee. However, good measurements were made in the regions of the high-latitude plasma sheet boundary and polar cap. In addition, EDI was not operated on spacecraft 4 during these orbits, so we present only data from spacecraft 1, 2, and 3. The periods of the Cluster perigee passes studied below are geomagnetically quiet to moderately active. $K_{P}$ is between $1+$ and $2+$ for the 8 $\mathrm{h}$ periods shown on each orbit. Wide band auroral images from the IMAGE WIC instrument show some substorm activity before and after the perigee passes, but relatively quiet arcs during the plasma sheet boundary crossings that are analyzed in detail below (private communication, Mende and Frey, 2001).

\subsection{February 2001}

Figure 4 shows an overview of EDI drift velocities for the 8-h orbit segment of Fig. 3, for Cluster spacecraft 1 (black), 2 (red), and 3 (green). The data are presented in a magnetic field oriented coordinate system, $\boldsymbol{X}_{\perp}, \boldsymbol{Y}_{\perp}, \boldsymbol{Z}_{\perp}$, defined for each spacecraft as follows:

$\boldsymbol{Z}= \pm \boldsymbol{B} / \boldsymbol{B}$

(sign chosen to have a positive $Z_{\mathrm{GSE}}$ component)

$\boldsymbol{X}_{\perp}=\boldsymbol{Y}_{\mathrm{GSE}} \times \boldsymbol{Z}$

$\boldsymbol{Y}_{\perp}=\boldsymbol{Z} \times \boldsymbol{X}_{\perp}$.

This system is well suited to display $\boldsymbol{E} \times \boldsymbol{B}$ (and other) drift velocities that are, by definition, perpendicular to the magnetic field. The $\boldsymbol{X}_{\perp}$ component represents drift within the plane perpendicular to $\boldsymbol{B}$ that is closest to the sunward direction, while the $\boldsymbol{Y}_{\perp}$ component is drift transverse to the Earth-Sun line in the duskward direction. For $\boldsymbol{B}$ parallel to

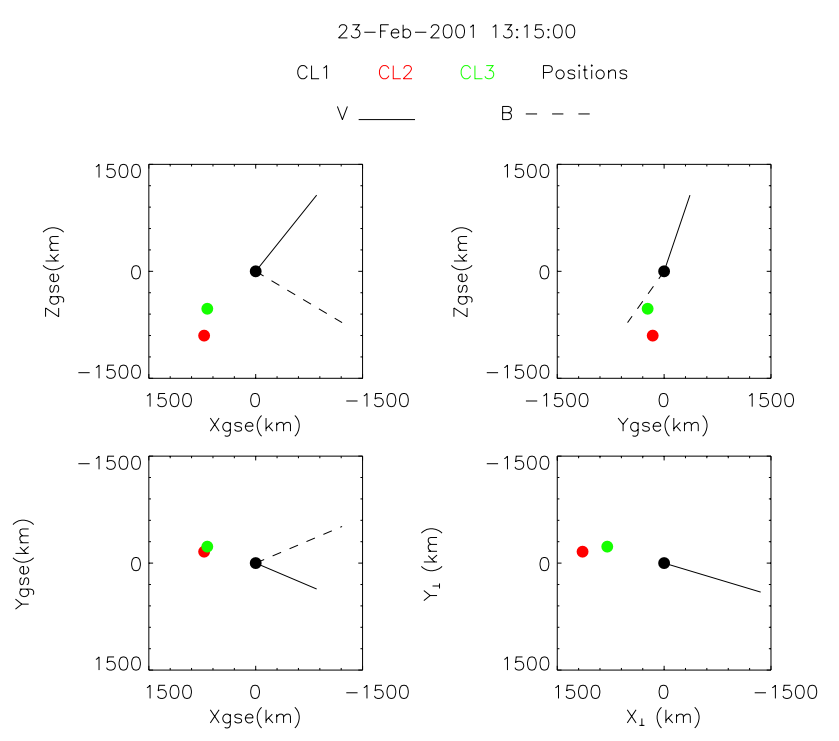

Fig. 6. Relative positions of spacecraft 1,2 and 3 at the time of crossing into the intense $1 \mathrm{keV}$ electron population of the southern plasma sheet on 23 February. Positions with respect to spacecraft 1 are represented by dots using the standard color assignments. Three panels show projections in the 3 principle GSE coordinate system planes. The lower right panel is the projection in the plane perpendicular to the magnetic field. $+\boldsymbol{X}_{\perp}$ is the direction closest to the Sun in this plane. The spacecraft velocity vector is shown by the solid line, scaled so that $5 \mathrm{~km} / \mathrm{s}$ corresponds to a value of 1500 on the $\mathrm{km}$ scale. The magnetic field unit vector projection is given by the dashed line.

$\boldsymbol{Z}_{\mathrm{GSE}}$, the $\boldsymbol{X}_{\perp}, \boldsymbol{Y}_{\perp}$ coordinates reduce to $\boldsymbol{X}_{\mathrm{GSE}}, \boldsymbol{Y}_{\mathrm{GSE}}$. For the remainder of the paper, we refer to the $\boldsymbol{X}_{\perp}-\boldsymbol{Y}_{\perp}$ plane as the " $\boldsymbol{B}_{\perp}$ plane".

The top two panels show the drift velocity components in the plane perpendicular to $\boldsymbol{B}$. The values for spacecraft 2 and 3 are offset for visibility by +10 and $-10 \mathrm{~km} / \mathrm{s}$, respectively, and the zero level for each spacecraft is indicated by the horizontal dashed line. The third and fourth panels show the magnitude of the velocity and its direction within the $\boldsymbol{B}_{\perp}$ plane with respect to $+\boldsymbol{X}_{\perp}$. Each point is the average drift velocity determined from all return beams in a $1 / 2$ spin. Details of the ground analysis process are given by Paschmann et al. (2001, this issue). The velocities are in an "inertial" frame, i.e. the contribution from spacecraft velocity has been subtracted. At the beginning of the interval, from about 10:30 to 12:00 UT, Cluster is moving across the southern polar cap at a geocentric distance of $6-7.5 R_{E}$. During this period, the $V X_{\perp}$ component shows an "anti-sunward" convection of about $10 \mathrm{~km} / \mathrm{s}$. Although the direction of $\boldsymbol{X}_{\perp}$ varies with the magnetic field orientation, the flow in the $\boldsymbol{X}_{\perp}$ direction is always towards the nightside. During this same period, the $V Y_{\perp}$ component of flow is smaller, oriented toward dusk at $<5 \mathrm{~km} / \mathrm{s}$.

As the satellites approach the plasma sheet (12:0012:40 UT), the anti-sunward flow (toward the nightside plasma sheet) and the duskward flow decrease, with both 


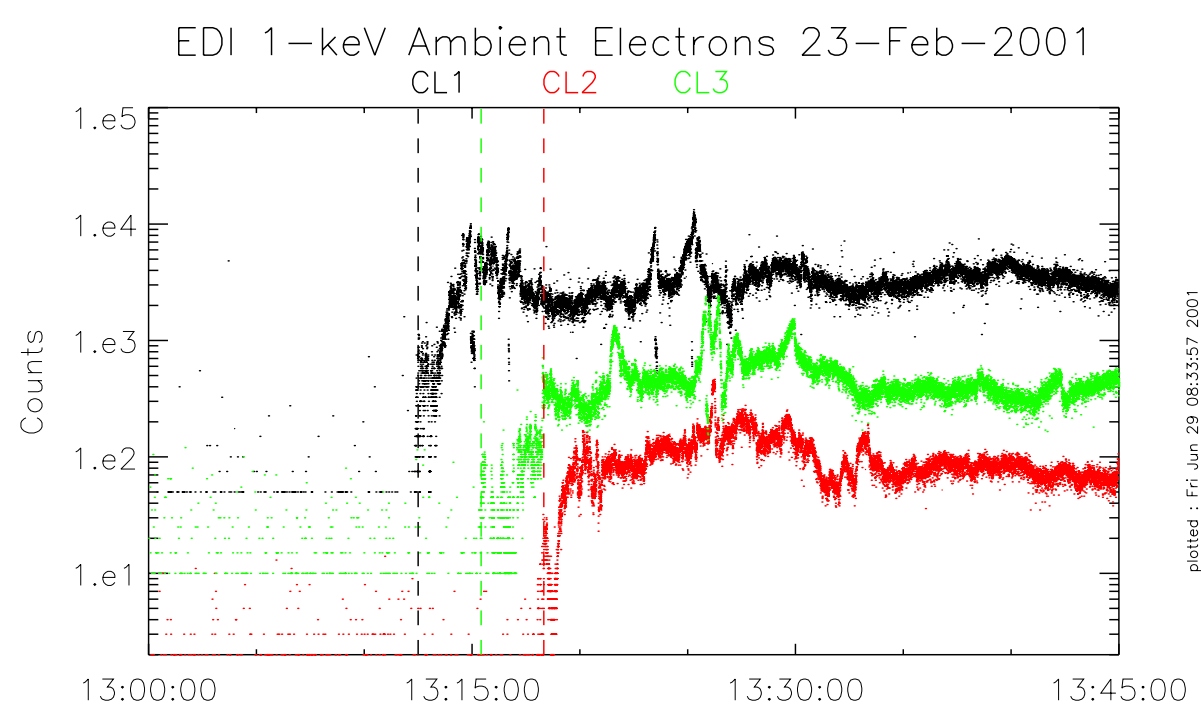

Fig. 7. EDI $1 \mathrm{keV}, 90^{\circ}$ pitch-angle ambient electron counts for spacecraft 1 , 2 , and 3 , using the same colors as in the other figures. Counts for spacecraft 1 and 3 are offset by factors of 25 and 5 , respectively. Count levels of 0 and 1 are clipped. Fiducial marks at the strong count intensifications seen by each spacecraft are plotted at 13:12:30, 13:15:25 and 13:18:20 UT. components becoming more variable as the $V X_{\perp}$ component reaches zero at about 12:50 UT. The vertical dashed lines at 13:12-13:18 mark the times of entry into the $1 \mathrm{keV}$ electron plasma sheet for each spacecraft, as discussed below in relation to Fig. 7. At this time, the $V Y_{\perp}$ component of flow shows a reversal, changing from duskward to dawnward.

Following entry into the plasma sheet, the flow velocity variability continues (seen in spacecraft 1) until operations are suspended through the equatorial perigee portion of the orbit. This variability is not fully resolved in the half-spin averaged data shown here.

In the northern hemisphere segment of the orbit, following the data gap at perigee, the flows are again highly variable until the satellites exit the plasma sheet at 16:10. At that time, the consistent anti-sunward flow resumes in the $V X_{\perp}$ component. The velocity of this "steady" anti-sunward flow in the northern hemisphere is immediately at $\sim 10 \mathrm{~km} / \mathrm{s}$, showing little sign of the slower flow transition near the plasma sheet boundary that was seen in the southern hemisphere.

Throughout this interval, the flows seen by the three satellites are in good agreement. At the time of the plasma sheet entry, the satellites are separated by distances of the order of $1000 \mathrm{~km}$, and times of about 1-2 min. As expected, the timing differences between the observed features are not readily apparent on the large time scale of Fig. 4.

We now look with higher time resolution at the southern hemisphere crossing from the polar cap into the plasma sheet. Figure 5 shows flow velocities in the same format as before for a 2-h interval containing this crossing. In the spacecraft 1 data, there is a distinct, qualitative change in the character of the velocities near the time of the black dashed line, from relatively smooth to much more variable, with fewer points. There are three contributions to this change. First and most important, the drift velocities are much more variable in the later region than in the preceding polar cap. A detailed examination of the beam triangulation plots (similar to those in Fig. 2) shows very well-defined intersection points over short intervals, but with a relatively large variation between many of the intervals. Thus, the variation seen following 13:12 UT is real, but is often not well resolved by the half-spin $(2 \mathrm{~s})$ intervals used in the figure. Rapid convection variations in the vicinity of the plasma sheet boundary are certainly not unexpected, and often continue to varying degrees into the equatorial regions (e.g. Quinn et al., 1999). Second, due to the flow variability, the ground software that determines the drift step, and thus the flow velocities from all beams within the averaging interval cannot, in many cases, obtain a sharplydefined result. Even though there are many return beams, the time variation of the drift velocity within the interval smears the beam intersection point. In these cases, a large value of chi-squared is obtained for the fit, and no point is plotted for that interval. This accounts for much of the sparser density of points in the velocity plots. We are adding to our software the capability to improve this resolution by using variable averaging intervals to better match the variations in the data. Third, in some instances rapid changes in the background electron fluxes, drift velocity, or magnetic field interfere with the ability of instrument's onboard tracking software to obtain sufficient beam returns within the averaging interval in order to yield an accurate measurement. It is interesting to note in the spacecraft 1 data that despite the increased variability, the flow oscillations that were visible on the polar cap side of the boundary continue following the crossing.

On the time scale of Fig. 5, there are noticeable differences in the spatial/temporal features seen on the three spacecraft. We explore these differences in the context of the satellites' relative positions and their times of entry into intense fluxes of $(1 \mathrm{keV})$ plasma sheet electrons, as displayed in Figs. 6 and 7 , respectively. Figure 6 shows the relative positions of spacecraft 1,2 , and 3 in four projections at 13:15 UT. Three panels show their position, relative to spacecraft 1 in the three principle GSE coordinate planes, while the fourth panel, in the lower right, is the projection in the $\boldsymbol{B}_{\perp}$ plane. Since electron motion along the field lines is very fast over 


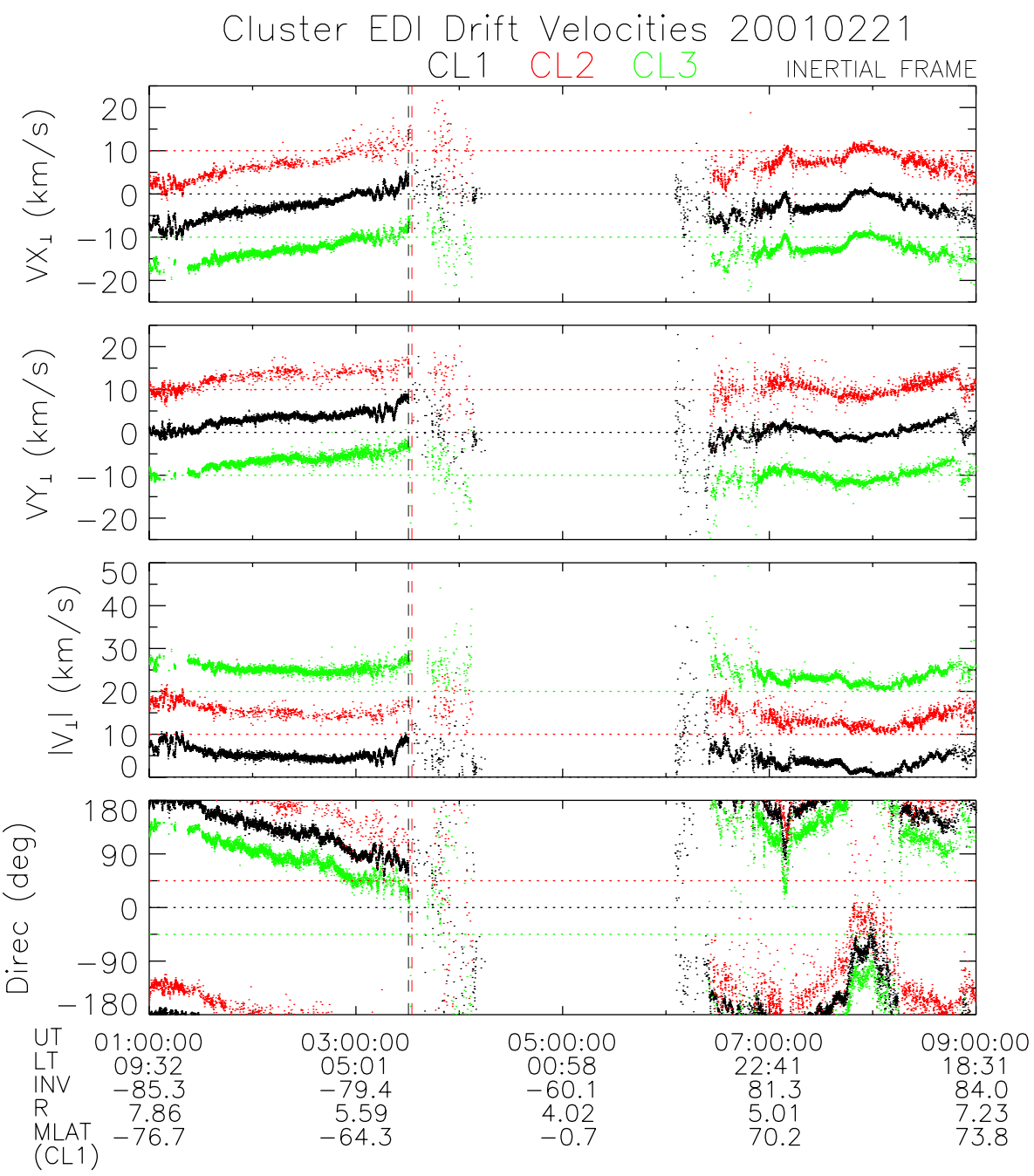

Fig. 8. Overview of drift velocities for 21 February in the same format as Fig. 4. Vertical dashed lines near 03:30 correspond to the first encounter with intense $1 \mathrm{keV}$ electrons, as marked in Fig. 11.

distances of the order of the satellite separations, one expects that the satellite and plasma motion projected in this plane will provide a good basis for interpreting variations in electron fluxes.

Similarly, in many cases, the drift velocity structures will also be ordered by structures perpendicular to the magnetic field. In addition to the satellite positions, each panel in Fig. 6 also shows the satellite velocity vector (solid line) and magnetic field unit vector (dashed line). The velocity vector is scaled so that $1500 \mathrm{~km}$ on the distance axis corresponds to $5 \mathrm{~km} / \mathrm{s}$. The satellites are moving nearly perpendicular to the magnetic field, toward the equatorial plane (compare with Fig. 3).

In the $\boldsymbol{B}_{\perp}$ plane, spacecraft 1 is leading, followed by spacecraft 3 and 2 at distances of about 800 and $1150 \mathrm{~km}$, respectively, along the spacecraft velocity vector. In this plane, the satellite velocity is primarily in the $-\boldsymbol{X}_{\perp}$ direction, corresponding to the motion approximately in the meridianal plane toward the equator, with very little dawn-dusk component. Thus, the velocity is approximately perpendicular to structures that are ordered by magnetic L-shells. With satellite velocities in the $\boldsymbol{B}_{\perp}$ plane of about $4.5 \mathrm{~km} / \mathrm{s}$, the separations given above correspond to time delays of approximately 3.0 and 4.3 min for spacecraft 3 and 2 with respect to spacecraft 1 .

Figure 7 shows the $1 \mathrm{keV}$ ambient electron fluxes measured by EDI as described in Sect. 2.3, using the standard colors for the three spacecraft. Each satellite detects a relatively sharp, $\times 100$ increase in flux, separated by a few minutes. Fiducial marks are plotted as vertical dashed lines at 13:12:30, 13:15:25, and 13:18:20 UT. The time differences between the spacecraft 1 encounter with the $1 \mathrm{keV}$ electron boundary and those of spacecraft 3 and 2 are 2.9 and $5.8 \mathrm{~min}$. Within the accuracy of placing the electron boundary crossings, the observed time delays are in good agreement with the times estimated above for crossing a static structure oriented along a magnetic field shell. The spacecraft 1-3 delay matches very closely the static boundary prediction ( 2.9 versus $3.0 \mathrm{~min}$ ). The longer spacecraft 1-2 delay differs more significantly from the prediction (5.8 versus $4.3 \mathrm{~min}$ ). The 


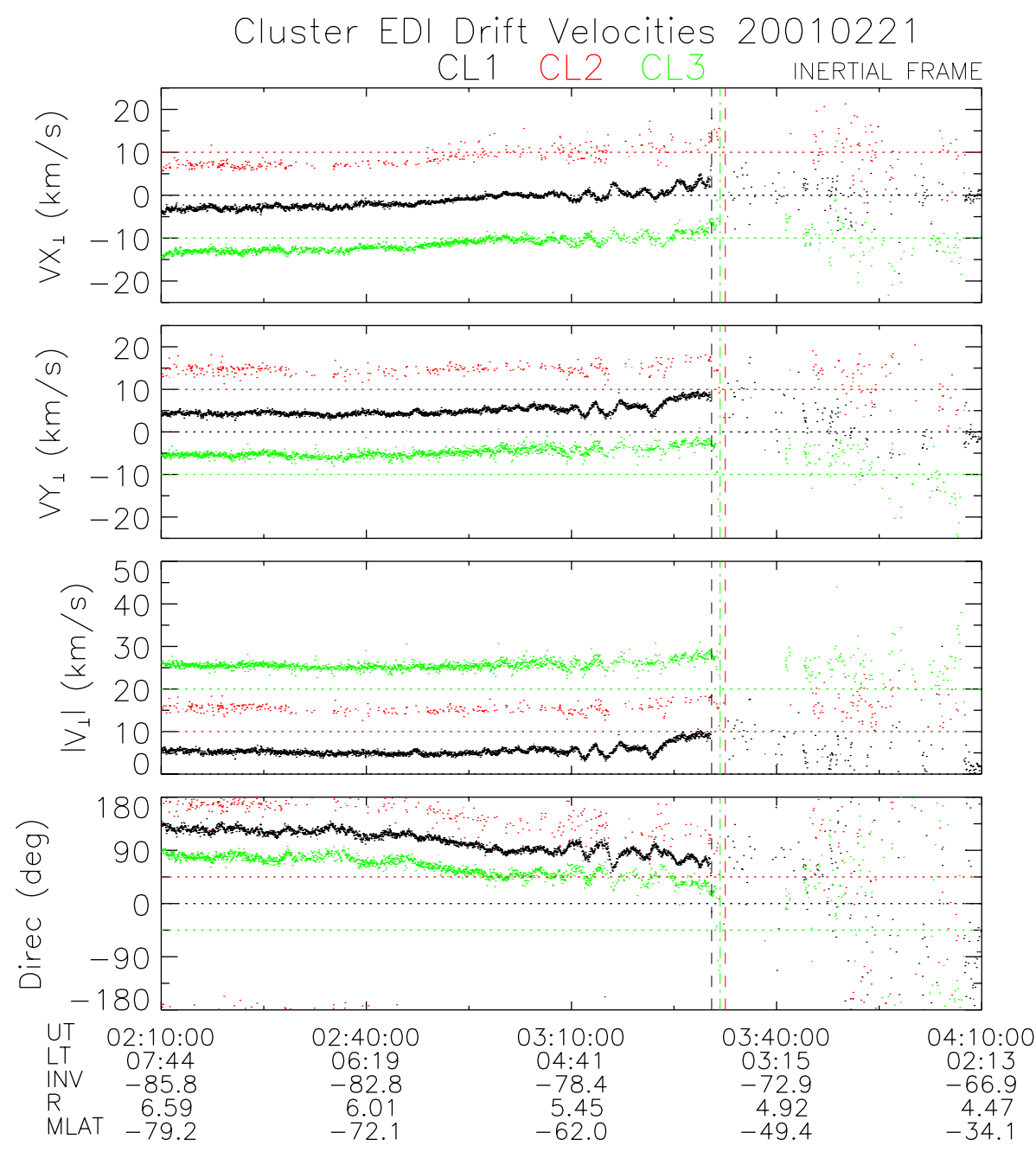

Fig. 9. Drift velocities during the 21 February southern hemisphere crossing from the polar cap into the high-latitude plasma sheet. Dashed markers plotted at the same times as those plotted in Fig. 8 and as the first set of markers in Fig. 11 indicate the satellites' encounters with the first 1-keV electron structure.

discrepancy between the two pairs of delays may be due to the limited accuracy of identifying and timing the boundary crossings using a single electron energy, or it might imply true boundary motion during the interval between the encounters by spacecraft 3 and 2 . A precise interpretation of the boundary motion and the many detailed variations seen in Fig. 7 requires electron spectral information that is not available in the standard EDI operations. From the $1 \mathrm{keV}$ data, we conclude that the velocity of the boundary during the several minutes of the satellite crossings is lower than, or at most comparable to, the spacecraft velocities.

The times of the intense $1 \mathrm{keV}$ electron encounters indicated in Fig. 7 are also marked in the drift velocity plots of Fig. 5. As seen on spacecraft 1, the $1 \mathrm{keV}$ electron encounter slightly precedes the convection reversal that is seen in the $\boldsymbol{Y}_{\perp}$ (dawn-dusk) component and in the flow direction.

\subsection{February 2001}

We now turn to the Cluster perigee pass on 21 February 2001, one orbit before the 23 February perigee discussed above. The satellites' orbits for this pass are very similar to that of 23 February, as shown in Fig. 3. Figure 8 shows an overview of EDI drift velocities for an 8-h period centered on perigee, in the same format as Fig. 4. The flows are qualitatively similar to those on 23 February in several ways. As the satellites approach perigee through the southern polar cap ( 01:00-02:30 UT), the largest component of the flow is anti-sunward. On both days, this $\boldsymbol{X}_{\perp}$ flow decreases while the duskward $\left(-\boldsymbol{Y}_{\perp}\right)$ component of flow picks up, although this change occurs over a longer interval on 21 February than on 23 February. As the satellites approach the auroral zone and plasma sheet, there is an interval of flow oscillations (beginning $\sim 03: 10 \mathrm{UT}$ ), followed by a sudden transition to highly variable flows and a reversal in the $V Y_{\perp}$ component from duskward to dawnward, again in common 


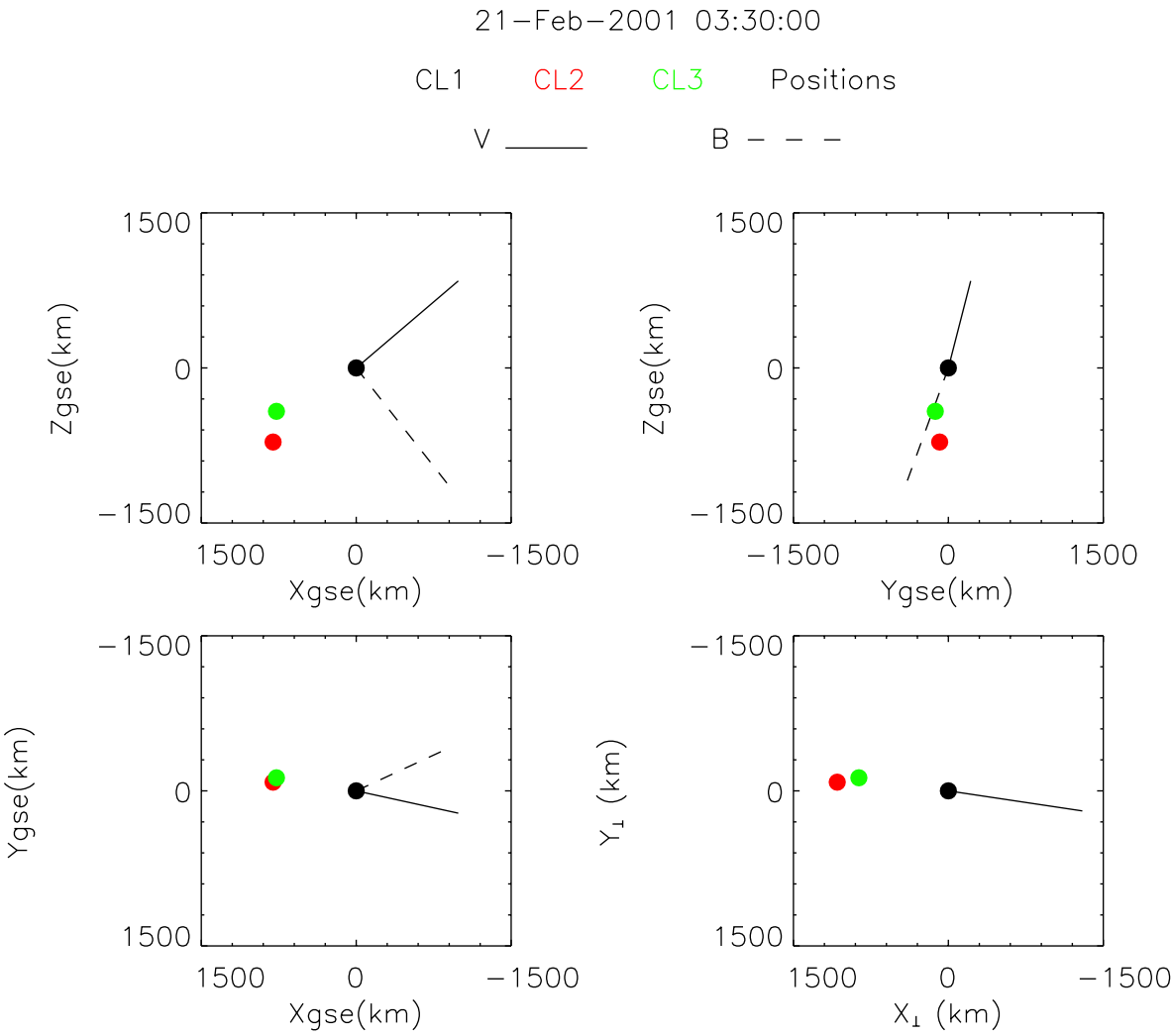

Fig. 10. Spacecraft 1, 2, and 3 relative positions, with velocity and magnetic field, at the time of the first encounter with intense $1 \mathrm{keV}$ electron counts (first markers in Fig. 11). Same format as Fig. 6.

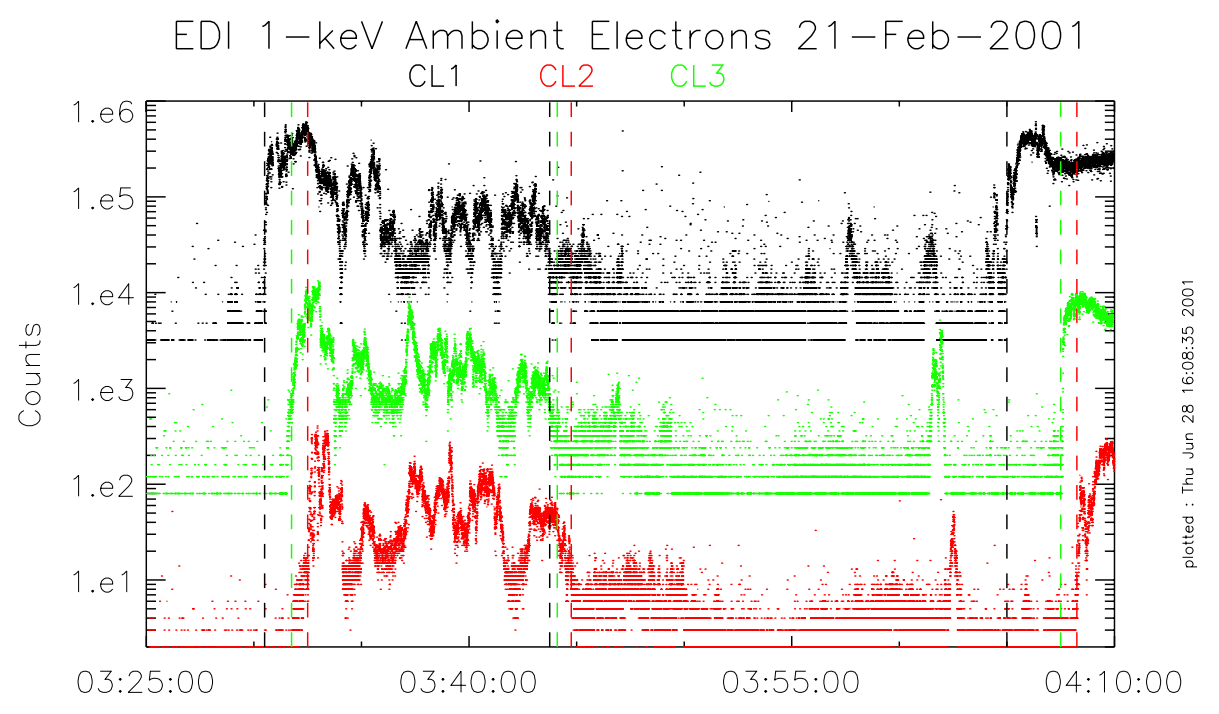

Fig. 11. $1 \mathrm{keV}$ ambient electron counts during the 21 February southern hemisphere crossing from the polar cap into the auroral zone/plasma sheet, whith the same format as Fig. 7. Counts for spacecraft 1 and 3 are offset by factors of 1600 and 40, respectively. The first region of high counts, from approximately 03:30-03:45 UT, is a crossing of a spatial structure, as seen by the fact that the three spacecraft enter and exit the feature in the same sequence. Horizontal bands near the bottom of each plot represent discrete count levels of 2 , 3,4 , etc. Count levels of 0 and 1 have been clipped. with 23 February. A higher time resolution view of the southern hemisphere crossing from the polar cap into the plasma sheet is presented in Figs. 9 to 11, which show the flow velocities, relative satellite positions, and $1-\mathrm{keV}$ electrons in the same formats, and with the same time scales, as the corresponding figures for 23 February. As before, spacecraft 1 is the lead spacecraft, followed in turn by spacecraft 3 and 2 . Figure 11 shows a more complicated and interesting structure at the electron boundary than was seen in the prior case. In particular, there are two distinct encounters with large 1-keV electron features. The three sets of dashed vertical lines indicate the entry and exit for the first feature, and the entry into the second. In the first feature, at about 03:30-03:45 UT, the satellite sequence across both the leading and trailing edges (first and second sets of vertical lines) is in the same order as their separation along the velocity vector in the $\boldsymbol{B}_{\perp}$ plane. This clearly establishes that the satellites are passing through an isolated feature, as opposed to having the boundary move across them and then back again (in which case, the order of exit would be opposite to the order of entry). Superim- 
posed on and between the two large electron features are many smaller features that are probably the result of both satellite motion and time variations.

The convection velocities in Fig. 9 show a pronounced change at the time of the encounter with the first electron structure in Fig. 11 (marked at 3:30:30, 3:31:45, and $3: 32: 30$ UT in both figures). As in the previous case, the velocities change from relatively smooth to highly variable, and they remain so throughout the rest of the interval, including the period between the isolated electron structure and the boundary that is crossed between 4:05:00 and 4:07:30 UT.

As in the previous case, the dawn-dusk velocity component in Fig. 9 reverses its sign in the vicinity of the plasma sheet boundary. However, in this instance, the reversal occurs over a longer interval, apparently occurring in the region between the isolated structure and the subsequent boundary crossing.

\section{Summary}

We have presented initial multi-spacecraft EDI measurements during two perigee passes from the polar cap into the high-latitude plasma sheet boundary region at midnight. The EDI technique provides accurate measurements of the full $\boldsymbol{E} \times \boldsymbol{B}$ drift velocity, independent of the orientation of the magnetic and electric fields. Despite the complexities of EDI's onboard beam-acquisition and tracking, the measurement is based upon simple geometry, yielding a well understood and reliable result. Throughout the two 8-h passes presented here, there is close agreement in the measurements by the three spacecraft on coarse time scales. As expected, there are strong differences between the satellites on time scales comparable to their orbital separation during their encounters with the plasma sheet boundary.

As the satellites approach the plasma sheet from the southern polar cap, the rather steady, primarily anti-sunward, flow decreases and becomes oscillatory. Near the plasma sheet boundary, the dawn-dusk component of the flow reverses. Within the plasma sheet, the flow velocities are much more variable, often showing significant differences between consecutive half-spin $(2 \mathrm{~s})$ measurements. However, on one of the two days presented (23 February), the flow oscillations are continuous across the boundary and well into the plasma sheet, indicating that the oscillation is rather largescale $\left(\sim 10^{\circ}\right.$ invariant latitude).

The multi-spacecraft observations of features in the electron drift velocities, together with EDI's measurement of $1 \mathrm{keV}$ ambient electrons, are consistent with boundary structures that are rather stable (moving at velocities less than the satellite velocities). Although the single-energy measurements presented cannot characterize the spectral and pitch angle structure of the electrons, they do provide a good proxy for plasma sheet entry as viewed from multiple satellites. In particular, on 23 February, the electron boundary is a sharp, single feature seen by all three satellites in sequence. In contrast, on 21 February, there is a separate electron structure poleward of the plasma sheet that is crossed by the satellites before entering the continous plasma sheet.

These initial observations illustrate that Cluster and EDI are well suited to investigations of the phenomena seen at the high-latitude plasma sheet boundary. We are beginning follow-on studies combining many different data sets to more fully characterize this rich region using the Cluster multipoint capabilities.

Acknowledgements. The authors thank the many people who have made the Electron Drift Instrument possible through years of development, testing, and operational support, in particular B. Austin, B. Briggs, J. Chan, M. Chutter, R. Frenzel, W. Göbel, J. Googins, D. Hallmark, W. Isaac, G. King, S. Longworth, K. Lynch, R. Maheu, F. Melzner, J. Needell, U. Pagel, P. Parigger, D. Simpson, K. Strickler and C. Young. We acknowledge the excellent support by the project team at ESTEC, in particular P. Escoubet and M. Fehringer, and the mission operations team at ESOC, in particular S. Matussi and M. Schmidt. We are grateful to A. Balogh and the Cluster FGM team for their close collaboration in providing the onboard magnetic field data used by EDI and for use of the FGM data in EDI ground analyses; and to N. Cornilleau-Wehrlin and the STAFF team for providing the onboard STAFF data. We thank M. Goldstein, L. Christensen, and D. Machi for their key support of Cluster and EDI at NASA; and the payload team at Dornier, in particular R. Nord. We acknowledge NASA SSCWeb for orbit plots. The authors remember fondly, and gratefully acknowledge, the profound contributions of N. Sckopke to EDI and Cluster, and the many pleasures brought by his presence and missed in his absence. This work was supported by NASA through grants NAS5-30744 and NAG5-9960, and by DLR through grants FKZ:50OC89043 and FKZ:50OC9705.

The Editor in Chief thanks M. Yamauchi an another referee for their help in evaluating this paper.

\section{References}

Baumjohann, W., Haerendel, G., and Melzner, F.: Magnetospheric convection observed between 06:00 and 21:00 LT: variations with $K p$, J. Geophys. Res., 90, 393, 1985.

Carlson, C. W., McFadden, J. P., Ergun, R. E., Temerin, M., Peria, W., Mozer, F. S., Klumpar, D. M., Shelley, E. G., Peterson, W. K., Moebius, E., Elphic, R., Strangeway, R., Cattell, C. and Pfaff, R.: FAST observations in the downward auroral current region: Energetic upgoing electron beams, parallel potential drops, and ion heating, Geophys. Res. Lett., 25, 2017k, 1998.

Cattell, C. A., Kim, M., Lin, R. P., and Mozer, F. S.: Observations of large electric fields near the plasmasheet boundary by ISEE-1, Geophys. Res. Lett., 9, 539, 1982.

Eastman, T. E., Frank, L. A., Peterson, W. K., and Lennartsson, W.: The plasma sheet boundary layer, J. Geophys. Res., 89, 1553, 1984.

Melzner, F., Metzner, G., and Antrack, D.: The Geos electron beam experiment, S 329, Space Science Instrumentation, 4, 45, 1978.

Mende, S. and Frey, H.: Image far ultraviolet instrument overview plots, http://sprg.ssl.berkeley.edu/image/, 2001.

Paschmann, G., Melzner, F., Frenzel, R., Vaith, H., Parrigger, P., Pagel, U., Bauer, O. H., Haerendel, G., Baumjohann W., Sckopke, N., Torbert, R. B., Briggs, B., Chan, J., Lynch, K., Morey, K., Quinn, J. M., Simpson, D., Young, C., McIlwain, C. E., Fil- 
lius, W., Kerr, S. S., Maheu, R., and Whipple, E. C.: The electron drift instrument for Cluster, Space Sci. Rev., 79, 233, 1997.

Paschmann, G., McIlwain, C. E., Quinn, J. M., Torbert, R. B., and Whipple, E. C.: The electron drift technique for measuring electric and magnetic fields, Measurement Techniques in Space Plasmas: Fields, Geophysical Monograph 103, AGU, 1998.

Paschmann, G., Sckopke, N., Vaith, H., Quinn, J. M., Bauer, O. H., Baumjohann,W., Fillius, W., Haerendel, G., Kerr, S. S., Kletzing, C. A., Lynch, K., McIlwain, C. E., Torbert, R. B., and Whipple, E. C.: EDI electron gyro time measurements on Equator-S, Ann. Geophysicae, 17, 1513, 1999.

Paschmann, G., Quinn, J. M., Torbert, R. B., Vaith, H., McIlwain, C. E., Haerendel, G., Bauer,O. H., Bauer, T., Baumjohann, W., Filliius, W., Foerster, M., Frey, S., Kerr, S. S., Kletzing, C. A., Puhl-Quinn, P. and Whipple, E. C.: The electron drift instrument on Cluster: overview of first results, Ann. Geophysicae, (this issue) 2001

Pederson, A., Cattell, C. A., Falthammer, C.-G., Knott, K., Lindqvist, P.-A., Manka, R. H., and Mozer, F. S.: Electric fields in the plasma sheet and plasma sheet boundary layer, J. Geophys. Res., 90, 1231, 1985.

Quinn, J. M., McIlwain, C. E., Haerendel, G., Melzner, F., Cauffman, D., Measurement of vector electric fields using electron test particles, Trans., AGU, EOS, 60, 918, 1979.

Quinn, J. M., Paschmann, G., Sckopke, N., Jordanova, V. K., Vaith,
H., Bauer, O. H., Baumjohann, W., Fillius, W., Haerendel, G., Kerr, S. S., Kletzing, C. A., Lynch, K., McIlwain, C. E., Torbert, R. B., and Whipple, E. C.: EDI convection measurements at 5$6 R_{E}$ in the post-midnight region, Ann. Geophysicae, 17, 12, 1503-1512, 1999.

Sauvaud, J.-A., Popescu, D., Delcourt, D. C., Parks, G. K., Brittnacher, M., Sergeev,V., Kovrazhkin, R. A., Mukai, T., and Kokubun, S.: Sporadic plasma sheet ion injections into the highaltitude auroral bulge: Satellite observations, J. Geophys. Res., 104, $2865,1999$.

Torbert, R. B. and Mozer, F. S.: Electrostatic shocks as the source of discrete auroral arcs, Geophys. Res. Lett., 5, 135, 1978.

Vaith, H., Frenzel, R., Paschmann, G., and Melzner, F.: Electron gyro time measurement technique for determining electric and magnetic fields, in: Measurement Techniques in Space Plasmas: Fields, (Eds) Pfaff, Borovsky, and Young, Geophysical Monograph, AGU, 103, 47, 1998.

Wygant, J. R., Keiling, A., Cattell, C. A., Johnson, M., Lysak, R. L., Temerin, M., Mozer, F. S., Kleting, C. A., Scudder, J. D., Peterson, W., Russell, C. T., Parks, G., Brittnacher, M., Germany, G., and Spann, J.: Polar spacecraft based comparisons of intense electric fields and Poynting flux near and within the plasma sheet-tail lobe boundary to UVI images: An energy source for the aurora, J. Geophys. Res., 18 675, 2000. 